\title{
Circadian Clock Protein PERIOD2 Suppresses the PI3K/Akt Pathway and Promotes Cisplatin Sensitivity in Ovarian Cancer
}

This article was published in the following Dove Press journal: Cancer Management and Research

\author{
Zhaoxia Wang' \\ Fengyan $\mathrm{Li}^{\prime}$ \\ Meiyan Wei' \\ Sanyuan Zhang' \\ Tong Wang $\mathbb{D}^{2}$
}

'Department of Gynecology, First Hospital of Shanxi Medical University, Taiyuan, Shanxi, People's Republic of China; ${ }^{2}$ Department of Health Statistics, School of Public Health, Shanxi Medical University, Taiyuan, Shanxi, People's Republic of China
Correspondence: Tong Wang

Department of Health Statistics, School of Public Health, Shanxi Medical University, Taiyuan, Shanxi, People's Republic of

China

Email Tongwang@sxmu.edu.cn

Sanyuan Zhang

Department of Gynecology, First Hospital of Shanxi Medical University, Taiyuan,

Shanxi, People's Republic of China

Tel +86-1383518II96

Email ffprofessor@126.com
Background: The mortality rate of ovarian cancer is the highest among gynecological tumors. The two factors leading to high mortality of ovarian cancer are late clinical stage and chemotherapy resistance. It is very important to reverse or intervene chemotherapy resistance. Abnormal circadian rhythm is related to the occurrence of tumor, and circadian clock protein PERIOD2 (PER2) acts as a tumor suppressor in cancer; however, little is known about its involvement in chemosensitivity.

Methods: This study aimed to investigate the role and underlying mechanisms of PER2 in ovarian cancer sensitivity to cisplatin. Overexpression and knockdown of PER2 were performed to explore its role in ovarian cancer cell sensitivity to cisplatin both in vitro and in vivo. The protein levels of PI3K, AKT, caspase 3, E-cadherin, and other drug resistancerelated molecules were determined in parental SKOV3 and SKOV3/DDP cells as well as in xenograft tumor tissues.

Results: Compared with parental cells, SKOV3/DDP cells had dramatically decreased PER2 expression, possibly due to hypermethylation in the PER2 promoter. PER2 overexpression significantly inhibited proliferation while promoting cisplatin-induced apoptosis in SKOV3 and SKOV3/DDP cells. In agreement, PER2-overexpressing SKOV3/DPP cells yielded significantly reduced tumor mass in cisplatin-treated mice compared with control cells. Mechanistically, PER2 overexpression remarkably reduced the protein amounts of PI3K, AKT, and MDR1 while increasing those of caspase 3 and E-cadherin in tumor tissues. Knockdown of PER2 exhibited opposite effects. PER2 overexpression also reduced the serum levels of TNF- $\alpha$ and IL- 6 in tumor-bearing mice before the initiation of cisplatin treatment.

Conclusion: This study suggests that loss of PER2 contributes to cisplatin resistance in SKOV3 cells, possibly by activating the PI3K/AKT pathway and EMT, inhibiting apoptosis, and promoting drug efflux and inflammatory responses. Overexpression of PER2 could reverse these alterations and sensitize both parental SKOV3 and SKOV3/DDP cells to cisplatin.

Keywords: circadian clock protein PERIOD2, epithelial ovarian cancer, cisplatin, phosphoinositide 3 Kinase, protein kinase B

\section{Background}

Ovarian cancer is one of the most common cancers worldwide, with the lowest 5 -year survival rate of $30-40 \%$ among all gynecologic malignancies. ${ }^{1,2}$ The standard treatment for ovarian cancer involves maximum debulking surgery followed by platinum- and taxane-based chemotherapy. ${ }^{3}$ Cisplatin belongs to platinum drugs 
that induce DNA damage by crosslinking with purine bases, leading to cancer cell apoptosis. ${ }^{4}$ It has been widely used to treat various cancers for over four decades, and remains the first-line treatment option for ovarian cancer in combination with taxanes. ${ }^{5,6}$ Although most patients initially respond to this regimen, nearly $75 \%$ cases relapse with chemoresistance that is responsible for treatment failure and $90 \%$ of ovarian cancer-related deaths. ${ }^{7}$ Thus, it is important to explore the molecular mechanisms underlying cisplatin resistance, in order to develop effective therapies for ovarian cancer.

PER2 is a circadian factor that participates in circadian rhythm regulation. ${ }^{8}$ Aberrant expression of circadian factors can disrupt the circadian clock, leading to the development and progression of many diseases, including cancer. $^{9}$ Recent studies have shown that PER2 acts as a tumor suppressor by promoting apoptosis while inhibiting proliferation and invasion in different cancer cells, including leukemia, osteosarcoma, and glioma cells. ${ }^{10-12}$ Our previous study demonstrated low PER2 expression in malignant tumors of the human ovary compared with benign lesions. Meanwhile, ectopic expression of PER2 suppresses xenograft tumor growth of SKOV3 cells in nude mice, ${ }^{13}$ suggesting a tumor-suppressive role for PER2 in ovarian cancer. However, the involvement of PER2 in cisplatin sensitivity remains unknown.

Chemoresistance in cancer involves multiple mechanisms, including apoptosis evasion, EMT, increased drug efflux, and enhanced inflammatory responses. ${ }^{3,14}$ The PI3K/Akt pathway plays a critical role in regulating cell proliferation, cell cycle, and cell apoptosis. ${ }^{15}$ Previous studies have linked PI3K/Akt pathway activation with cisplatin resistance. ${ }^{16}$ Hyperactivated PI3K/AKT pathway due to mutations and other mechanisms plays an important role in mediating anti-apoptotic activity in chemoresistance. ${ }^{17}$ EMT, characterized by loss of E-cadherin, is a process in which epithelial cells acquire mesenchymal features by losing their cell-cell adhesive properties and gaining migratory and invasive potential. ${ }^{18}$ EMT contributes to cisplatin resistance in various malignancies, including ovarian cancer. ${ }^{19}$ Multidrug resistance 1 (MDR1) and MDR1related protein 1 (MRP1) encode transporters that export drugs from cells, leading to reduced intracellular drug accumulation. ${ }^{20}$ Cisplatin induces MDR1 transcription in ovarian cancer cell lines. ${ }^{21}$ Meanwhile, N-linked glycosylation of MRP1 remarkably reduces cisplatin accumulation in ovarian cancer cells, resulting in cisplatin resistance. ${ }^{22}$ In addition, ovarian cancer initiation and progression involve inflammation. ${ }^{23}$ Inflammatory mediators such as IL-6 and IL-8 promote drug resistance by increasing drug efflux and reducing apoptosis. ${ }^{24}$

In the present study, we investigated the role and underlying mechanisms of PER2 in ovarian cancer cell sensitivity to cisplatin both in vitro and in vivo. The results suggest that loss of PER2 in SKOV3/DDP cells contributes to cisplatin resistance, and its overexpression may serve as a potential therapeutic strategy for overcoming cisplatin resistance in ovarian cancer.

\section{Methods}

\section{Cell Lines and Culture}

SKOV3 cells were purchased from the American Type Culture Collection (Manassas, VA, USA), and maintained in RPMI 1640 (Hyclone, GE Healthcare Life Sciences, Logan, UT, USA), supplemented with $10 \%$ fetal bovine serum (Gibco FBS, New Zealand), 1\% penicillin, and 1\% streptomycin in a $5 \% \mathrm{CO}_{2}$ incubator at $37{ }^{\circ} \mathrm{C}$ with saturated humidity.

SKOV3/DDP cells were obtained from Shanghai YaJi Biological Co., Ltd (China), and maintained in RPMI 1640 supplemented with $10 \%$ FBS, $1 \%$ penicillin, $1 \%$ streptomycin, and $1 \mu \mathrm{g} / \mathrm{mL}$ of cisplatin.

\section{Plasmid Construction and Transfection}

For PER2 overexpression, human PER2 cDNA was cloned into the pcDNA3.1 vector (GeneCreate Biological Co., Ltd, China). The empty vector was used as a negative control. PER2 knockdown was performed by transfection with siRNA against PER2 (si-PER2; 5'- GCUGGUCCAGCUU CAUCAATT-3'), using scrambled siRNA as a negative control. SKOV3 cells were transfected with the PER2overexpressing vector, si-PER2, or corresponding negative controls with Lipofectamine ${ }^{\circledR} 2000$ (Invitrogen, Carlsbad, CA, USA).

\section{Tumor Xenograft Model}

Ten BALB/c nude mice (5-week old, all female) were purchased from Beijing Huafukang biotechnology co., Ltd (Beijing, China), and maintained under specific pathogenfree conditions. All animal care and experimental procedures were approved by the Ethics Committee of Shanxi Medical University (Shanxi, China). The animal experiments followed the Guidelines for Proper Conduct of Animal Experiments of Shanxi Medical University. After 1 week of acclimation, mice were randomly divided into 2 
groups ( $\mathrm{n}=5$ /group). SKOV3 cells $\left(1 \times 10^{7}\right.$ in $0.2 \mathrm{~mL}$ of serum-free RPMI 1640) transfected with the empty or PER2-overexpressing vector were inoculated into the rightback of each mouse by subcutaneous injection, plasmid $(1.5 \mathrm{mg} / \mathrm{kg})$ was mixed with transfection reagent, and then was intratumor injected in PER2+ group every Monday and Thursday. ${ }^{25}$ Tumor formation was monitored every other day. Cisplatin ( $3 \mathrm{mg} / \mathrm{kg}$, twice a week) was administered by intraperitoneal injection at 2 weeks (Tuesday and Friday) after inoculation when the tumors became visible. Tumors were measured every week using a digital caliper, and tumor volume $(\mathrm{V})$ was derived by the following equation: $\mathrm{V}=\left(\right.$ length $\times$ width $\left.^{2}\right) / 2$. Mice were sacrificed at 3 weeks after cisplatin treatment initiation. Tumors were immediately removed and stored at $-80^{\circ} \mathrm{C}$ until use.

\section{Bisulfite-Sequencing PCR}

Genomic DNA was isolated from SKOV3 or SKOV3/DDP cells with a genomic DNA extraction kit (Aidlab, Beijing, China). Bisulfite modification of genomic DNA was performed with an EZ DNA Methylation-GoldTM kit (Zymo Research, Irvine, CA, USA), followed by PCR amplification with the primer sets shown in Table 1. PCR products were purified and inserted into the pClone007 vector (Tsingke Biological Technology, Beijing, China) following the manufacturer's instructions. DNA methylation status was analyzed by sequencing.

\section{Cell Viability Assay by Cell Counting Kit-8 (CCK8)}

Parental SKOV3 and SKOV3/DDP cells were seeded in 96-well plates $\left(4 \times 10^{3}\right.$ cells per well $)$ and incubated overnight, followed by cisplatin $(5 \mu \mathrm{g} / \mathrm{mL})$ treatment. Then, $10 \mu \mathrm{L}$ CCK 8 solution was added to each well after 0,24 , and $48 \mathrm{~h}$ of cisplatin treatment, respectively, followed by incubation in the dark for $1-4 \mathrm{~h}$. Absorbance (OD value) was determined at $450 \mathrm{~nm}$ on a SpectraMax 190 microplate reader (Marshall Scientific, Hampton, NH, USA).

\section{Flow Cytometry}

Parental SKOV3 or SKOV3/DDP cells were transfected with the PER2-overexpressing vector, si-PER2, or corresponding negative controls, followed by cisplatin treatment $(5 \mu \mathrm{g} / \mathrm{mL})$. Cells were harvested by trypsinization, and washed twice with ice-cold phosphate-buffered saline (PBS) by centrifugation at $1000 \mathrm{rpm}$ for $5 \mathrm{~min}$. After resuspension in $100 \mu \mathrm{L}$ binding buffer, cells were stained with Annexin-V-FITC/propidium iodide solution (Solarbio, Beijing, China) following the manufacturer's instructions. Apoptosis was analyzed on an Attune NxT flow cytometer (Thermo, Waltham, MA, USA).

\section{Quantitative Real-Time PCR}

Total RNA was isolated with TriPure (Roche, USA), and reversely transcribed using M-MLV reverse transcriptase (ELK Biotechnology, Wuhan, Hubei, China). Amplification was performed with EnTurbo $^{\mathrm{TM}}$ SYBR Green PCR SuperMix on a StepOne ${ }^{\mathrm{TM}}$ Real-Time PCR instrument (Life Technologies, Carlsbad, CA, USA). Primers are shown in Table 1. $\beta$-actin was used as an internal control. Relative gene expression was quantified by the $2^{-\Delta \Delta \mathrm{Ct}}$ method.

\section{Western Blot}

SKOV3 cells and tumor tissues were lysed and homogenized, respectively, in lysis buffer on ice. The lysates were collected by centrifugation at $16,000 \mathrm{~g}$ for $10 \mathrm{~min}$ before heating at $95^{\circ} \mathrm{C}$ for $5 \mathrm{~min}$ in loading buffer. Samples were separated by $10 \%$ SDS-PAGE and transferred onto polyvinylidene difluoride membranes, followed by $1 \mathrm{~h}$ of blocking with $5 \%$ fat-free milk in Tris-buffered saline containing $0.1 \%$ Tween 20 (TBS$\mathrm{T})$. The membranes were incubated with primary antibodies raised against PER2 (1:1000; Proteintech, Rosemont, IL, USA), AKT (1:2000; Proteintech), PI3K (1:2000; Cell Signaling Technology, Danvers, MA, USA), caspase3 (1:1000; Proteintech), E-cadherin (1:1000; Proteintech), MDR1 (1:1000; Proteintech), MRP1 (1:1000; Abcam), DYRK2 (1:1000, Abcam), and GAPDH (1:10,000; Abcam, Cambridge, UK), respectively, overnight at $4^{\circ} \mathrm{C}$. The membranes were washed with TBS-T 3 times, and incubated with horseradish peroxidase-conjugated secondary antibodies for 1

Table I Primers

\begin{tabular}{|l|l|l|}
\hline Gene & Forward $\left(\mathbf{5}^{\prime} \mathbf{-} \mathbf{3}^{\prime} \mathbf{)}\right.$ & Reverse (5'-3') \\
\hline Actin & GTCCACCGCAAATGCTTCTA & TGCTGTCACCTTCACCGTTC \\
PER2 & GGACCAACAAGGTGCTGAGAGT & TGAGGTAGGTAGCCCAGGAGAG \\
Methylated-PER2 & GGGAGGGGTGTCTCACATCTGTAAT & GGTTGCAGTGAGCCGAGATCATGCCA \\
\hline
\end{tabular}

Abbreviation: PER2, PERIOD 2. 
$\mathrm{h}$ at room temperature. After TBS-T washes, chemiluminescent signals were detected on X-ray films and quantified with Image-J (NIH, Bethesda, MD, USA).

\section{Immunohistochemistry Stain}

Sections of paraffin-embedded tissue were subjected to immunohistochemical staining. We randomly selected at least 3 fields of view for each slice in each group. The CD43 antibody (14,486-1-AP15940-1-AP, Proteintech Group, Inc. USA) was used at 1:300. Sections were incubated with primary antibodies $\left(4{ }^{\circ} \mathrm{C}\right.$, overnight), followed by incubation of the poly-horseradish peroxidase anti- rabbit secondary antibody $\left(37^{\circ} \mathrm{C}, 20 \mathrm{~min}\right.$, dilution ratio 1:200; A24531, Aspen. China). Next, diaminobenzidine (DAB) was used to visualize the target proteins. Moreover, sections were counterstained with hematoxylin, dehydrated, and mounted. The expression of CD43 were evaluated using Image-pro plus 6.0 (Media Cybernetics, Inc., Rockville, MD, USA).

\section{ELISA}

Blood samples were obtained from mouse tail vein at 1 and 2 weeks after tumor cell implantation, respectively. The serum levels of TNF $\alpha$ and IL-6 were measured with
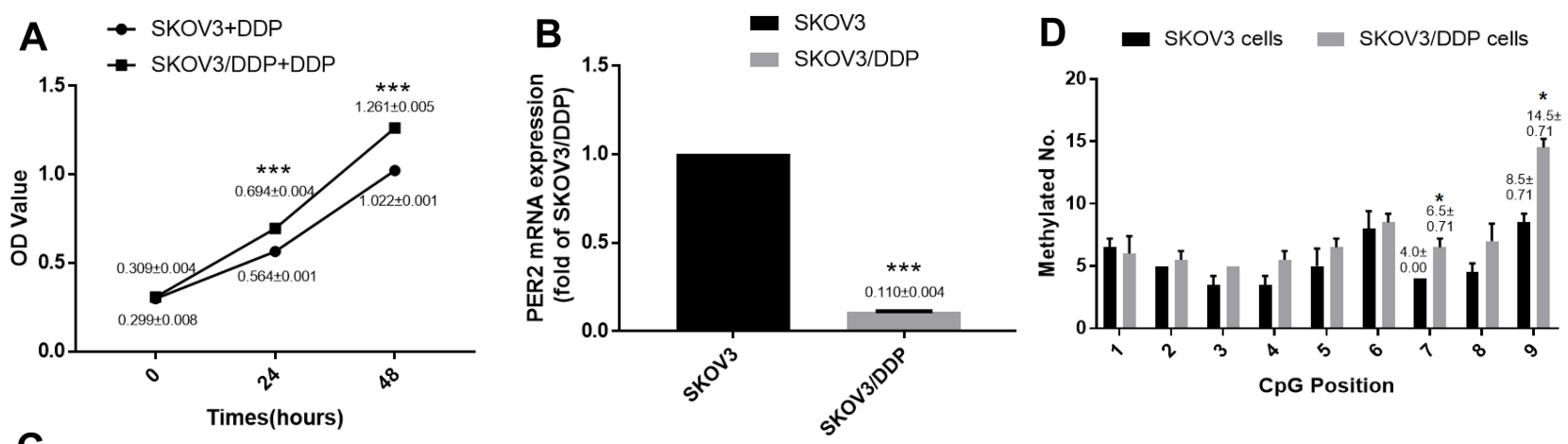

C

Methylation
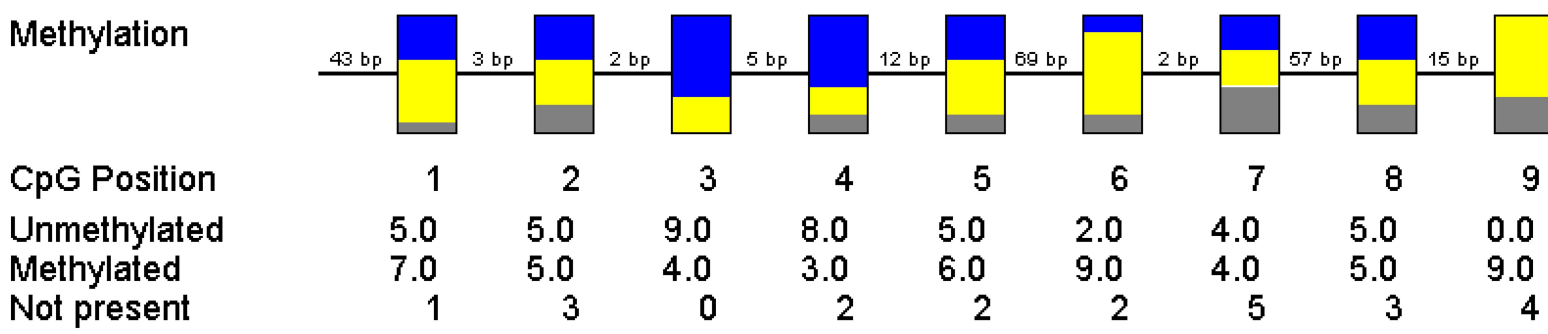

SKOV3 cell

Legend: $\quad \square$ unmethylated $\square$ methylated $\square$ not present

Methylation
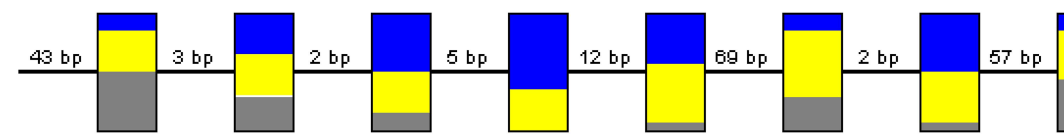

CpG Position
Unmethylated
Methylated
Not present

$\begin{array}{rrr}1 & 2 & 3 \\ 2.0 & 5.0 & 7.0 \\ 5.0 & 5.0 & 5.0 \\ 7 & 4 & 2\end{array}$

$\begin{array}{rr}3 & 4 \\ .0 & 9.0 \\ 0 & 5.0 \\ 2 & 0\end{array}$

45

6 7 8 $15 \mathrm{bp} \quad 17 \mathrm{bp}$

SKOV3/DDP cell

Legend: $\square$ unmethylated $\square$ methylated $\square$ not present

Figure I (A) Low expression of circadian clock protein PER2 in SKOV3/DDP cells. SKOV3 and SKOV3/DDP cells were treated with cisplatin ( $5 \mu \mathrm{g} / \mathrm{mL})$, performed by CCK8 , ***p $<0.001$ vs SKOV3 cells. (B) qRT-PCR was performed to detect endogenous mRNA expression of PER2 in SKOV3 and SKOV3/DDP cells. **** $<0.001$ vs SKOV 3 cells. (C) Bisulfite-sequencing PCR was performed to detect the DNA methylation status of CPG islands within the PER2 promoter. (D) The methylated numbers of CPG islands in the PER2 promoter, about No.7 and No.9 sites, was significantly higher than in SKOV3 cells. ${ }^{p} p<0.05$ vs SKOV3/DDP cells. 
ELISA kits (Jiangsu Kete Organism Co Ltd., 96T) following the manufacturer's instructions.

\section{Statistical Analysis}

All data were expressed as the mean with standard errors. For each cell line, comparison was performed using Oneway ANOVA or two-way ANOVA among more than two groups. Data were visualized by the GraphPad Prism7 software. For all the presented data, three independent experiments were performed to acquire the average results, $\mathrm{p}<0.05$ considered statistically significant.

\section{Results}

\section{Loss of PER2 in SKOV3/DDP Cells}

Our previous findings suggest a tumor suppressor role for PER2 in ovarian cancer. To further explore whether PER2 is associated with cisplatin resistance in ovarian cancer, we determined the mRNA expression of PER2 in parental SKOV3 and SKOV3/DDP cells. As shown in Figure 1A, SKOV3/DDP cells exhibited cisplatin resistance, as evidenced by significantly enhanced cell viability in the presence of cisplatin compared with parental cells. Then, qRT-PCR revealed that PER2 mRNA levels were dramatically decreased in SKOV3/DDP cells compared with that parental cells (Figure 1B), suggesting a possible relation between PER2 downregulation and cisplatin resistance in SKOV3 cells. To explore the possible mechanism underlying loss of PER2 in SKOV3/DDP cells, refer to
Hamilton method, ${ }^{9}$ we detected the DNA methylation status of $\mathrm{CpG}$ islands within the PER2 promoter. As shown in Figure 1C and in D, PER2 had significantly increased promoter methylation at nine $\mathrm{CpG}$ sites in SKOV3/DDP cells compared with parental cells, especially No.7 and No.9 sites which had significant difference, suggesting that $\mathrm{CpG}$ promoter methylation possibly contributed to loss of PER2 in SKOV3/DDP cells.

\section{PER2 Increases the Sensitivity of SKOV3 and SKOV3/DDP Cells to Cisplatin}

To examine the role of PER2 in cisplatin sensitivity, we performed respectively function assays for si-PER2 and overexpression plasmid transfected into SKOV3 and SKOV3/DDP cells in the presence of cisplatin. Figure $2 \mathrm{~A}-\mathrm{C}$ shows that the expression of PER2 was more lower or higher in SKOV3/DDP cells than SKOV3 by CCK-8, $* p<0.05, * * p<0.01, * * * p<0.001$. Then, cell viability assay showed that PER2 knockdown significantly promoted whereas its overexpression inhibited, the proliferation in both SKOV3 and SKOV3/DDP cells (Figure 3A and $\mathrm{B})$. Consistent results were observed for cell apoptosis by flow cytometry measurement (Figure 3C and D), which were flow chart as Figure 3C1-4 and D1-4, compared with the proliferation of cell viability. These findings suggested that PER2 inhibited cell proliferation while promoting cell apoptosis, thus increasing the sensitivity of both parental SKOV3 and SKOV3/DDP cells to cisplatin.
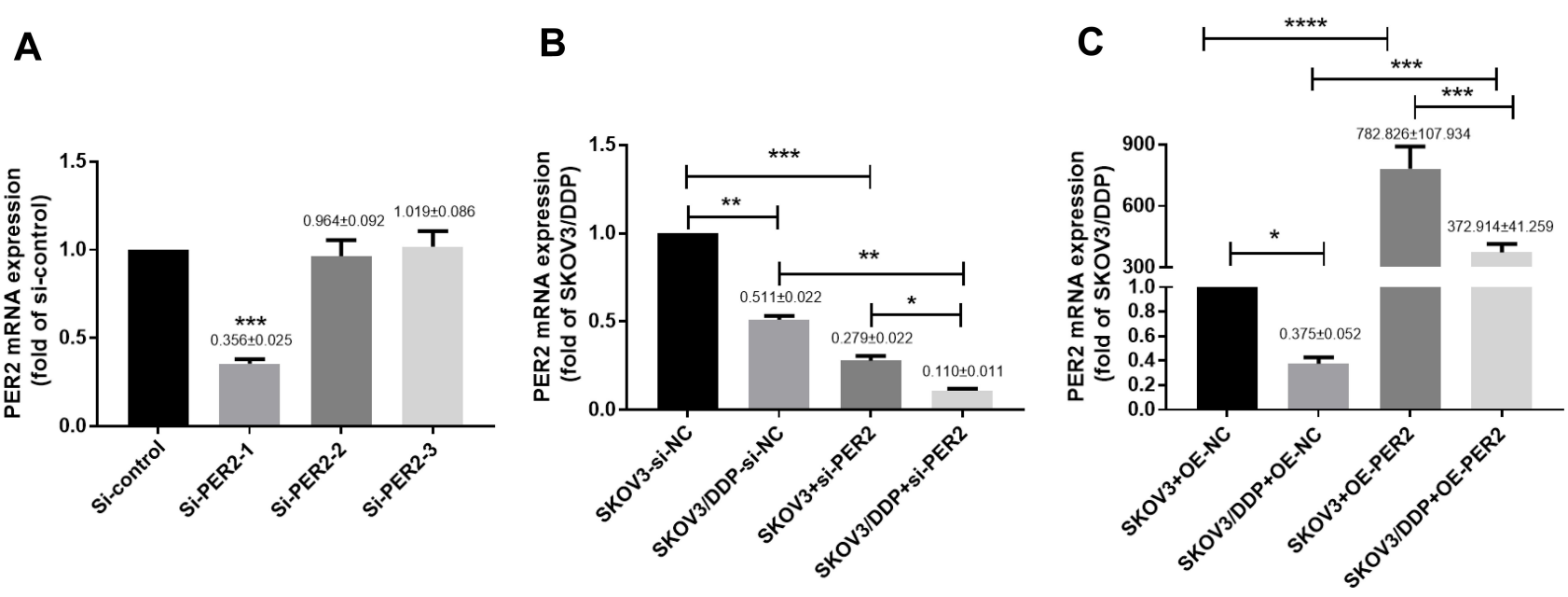

Figure 2 (A) Expression of PER2 in SKOV3 and SKOV3/DDP cells under cisplatin. SKOV3 cells were transfected with si-PER2 or si-control after 48 h, expression of PER2 mensurated by qRT-PCR, $\beta$-actin used as an internal control. ${ }^{* * *} p<0.001$ vs si-control in SKOV 3 cells. (B) SKOV3 and SKOV3/DDP cells were transfected with si-PER2 after $48 \mathrm{~h}$. The expression of PER2 was performed by qRT-PCR, with $\beta$-actin as an internal control. **p $<0.01$, ***p $<0.001$, SKOV3/DDP-si-NC or SKOV3-si-PER2 vs SKOV3-siNC. ${ }^{* *} p<0.01$, SKOV3/DDP-si-PER2 vs SKOV3/DDP-si-NC. ${ }^{*} p<0.05$, SKOV3/DDP-si-PER2 vs SKOV3-si-PER2. (C) The PER2-overexpressing vector, or negative control transfected to the SKOV3/DDP and parental cells, to performed the expression of PER2 by qRT-PCR. *p < 0.05, ****p < 0.000I, SKOV3/DDP-OE-NC or SKOV3-OE-PER2 vs SKOV3-OE-NC; ***p < 0.00I, SKOV3/DDP-OE-PER2 vs SKOV3/DDP-OE-NC; ***p < 0.00I, SKOV3/DDP-OE-PER2 vs SKOV3-OE-PER2. 


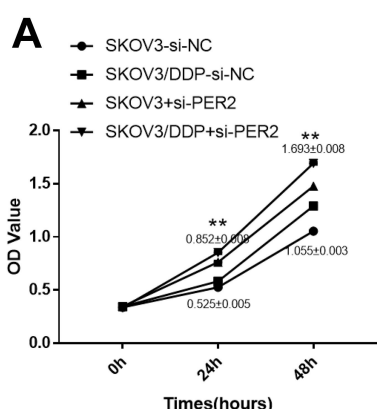

C1

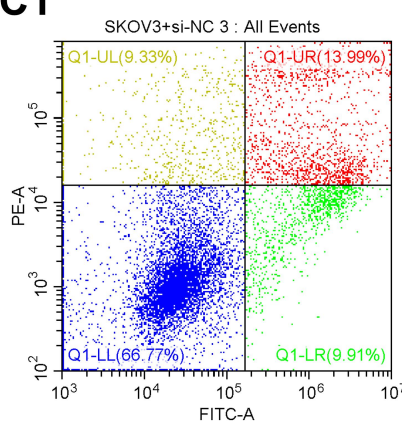

D1

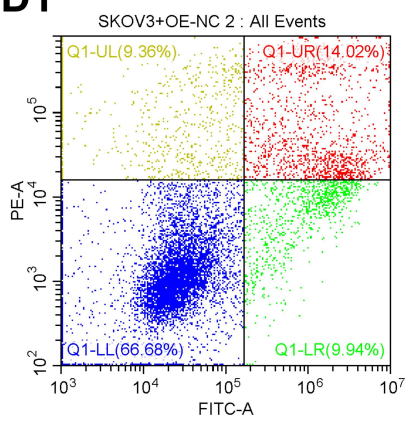

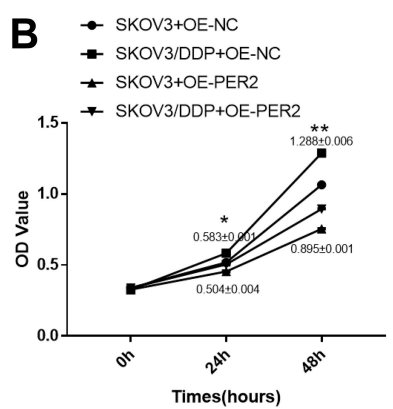

C2

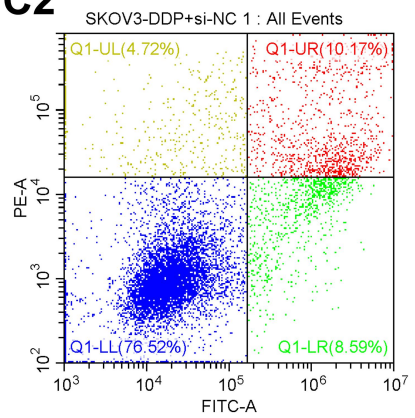

D2

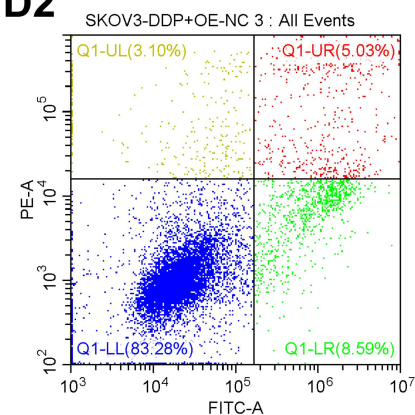

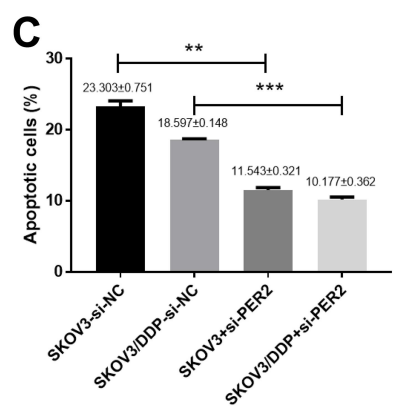

C3

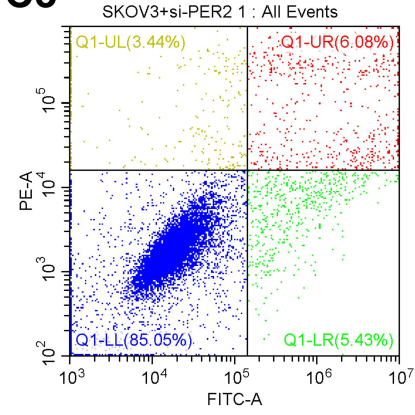

D3

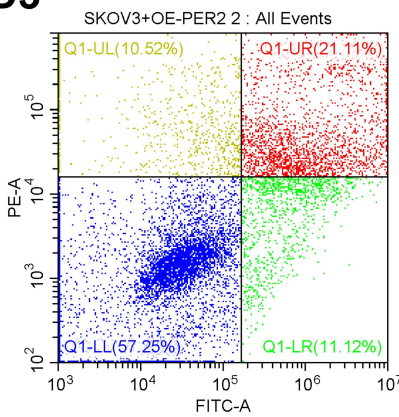

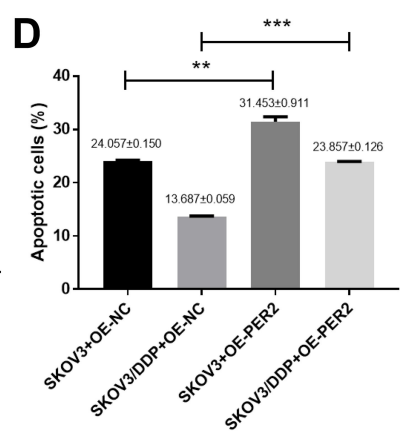

C4

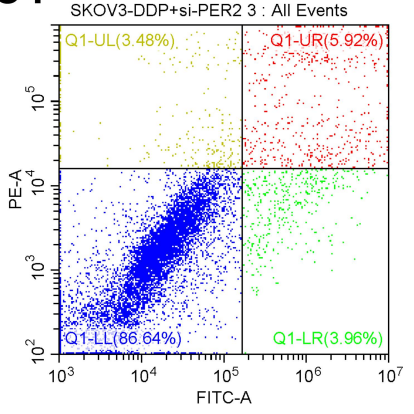

D4

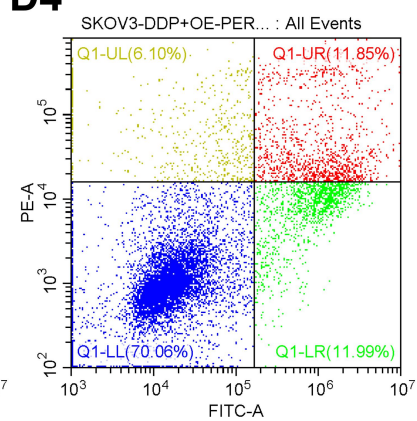

Figure 3 PER2 increases the sensitivity of SKOV3 and SKOV3/DDP cells to cisplatin. SKOV3 and SKOV3/DDP cells were transfected with si-RNA or overexpressing vector for PER2, followed by $5 \mu \mathrm{g} / \mathrm{mL}$ cisplatin treatment, vs si-NC or empty vector. (A-B) Cell viability was determined at different times after treatment by CCK-8, respectively. OD value was determined at $450 \mathrm{~nm}$ on a microplate reader. ${ }^{\mathrm{p}} \mathrm{p}<0.05$, SKOV3-si-PER2 vs SKOV3-si-NC and SKOV3/DDP-si-NC; **p $<0.01$, SKOV3/DDP-si-PER2 vs SKOV3-si-NC and SKOV3/DDP-si-NC; *p < 0.05, SKOV3/DDP-OE-NC vs SKOV3/DDP-OE-PER2; **p < 0.0I, SKOV3/DDP-OE-NC vs SKOV3-OE-NC; **p< 0.01, SKOV3OE-NC vs SKOV3/DDP-OE-PER2; **p $<0.01$, SKOV3/DDP-OE-PER2 vs SKOV3-OE-PER2 $n=6$. (C-D) The percentages of apoptotic cells were determined after treatment $48 \mathrm{~h}$ by Flow Cytometry. $* *$ p < 0.0I, SKOV3-si-PER2 vs SKOV3-si-NC; ***p < 0.00I, SKOV3/DDP-si-PER2 vs SKOV3/DDP-si-NC; **p < 0.0I, SKOV3-OE-PER2 vs SKOV3OE-NC; ***p < 0.00I, SKOV3/DDP-OE-PER2 vs SKOV3/DDP-OE-NC. (CI-C4) The apoptosis images of following groups respectively: (CI) SKOV3+si-NC, (C2) SKOV3/ DDP+si-NC, (C3) SKOV3+si-PER2, (C4) SKOV3/DDP+si-PER2. (DI-D4) The apoptosis images of following groups respectively: (DI) SKOV3+OE-NC, (D2) SKOV3/DDP +OE-NC, (D3) SKOV3+OE-PER2, (D4) SKOV3/DDP+OE-PER2. Data are mean $\pm S D, n=3$.

\section{PER2 Suppresses the PI3K/Akt Pathway and Regulates Genes Involved in Cell Apoptosis, EMT, and Drug Transportation}

Cisplatin resistance involves multiple mechanisms, including activation of the PI3K/Akt pathway, EMT, apoptosis evasion, and dysregulated drug transportation. ${ }^{16,19,25}$ To explore the possible mechanisms underlying the beneficial role of PER2 in cisplatin sensitivity, we quantitated the expression levels of representative molecules involved in these processes. As shown in Figure 4A, SKOV3/DDP cells had significantly increased protein levels of PI3K and Akt compared with parental SKOV3 cells, suggesting an activated PI3K/Akt pathway in SKOV3/DDP cells. Loss of caspase-3 and E-cadherin suggested inhibited apoptosis and enhanced EMT in SKOV3/DDP cells. The gray value of Western blot were as shown in Figure 4A1.

After PER2 was silenced, the expression of Akt, PI3K and MDR1 in SKOV3 and SKOV3/DDP was significantly up-regulated, whereas Caspase 3 and E-cadherin were significantly down regulated. However, after overexpression of PER2, the expression of Akt, PI3K and MDR1 in SKOV3 and SKOV3/DDP cells were significantly down regulated, caspase 3 and E-cadherin were significantly up-regulated 

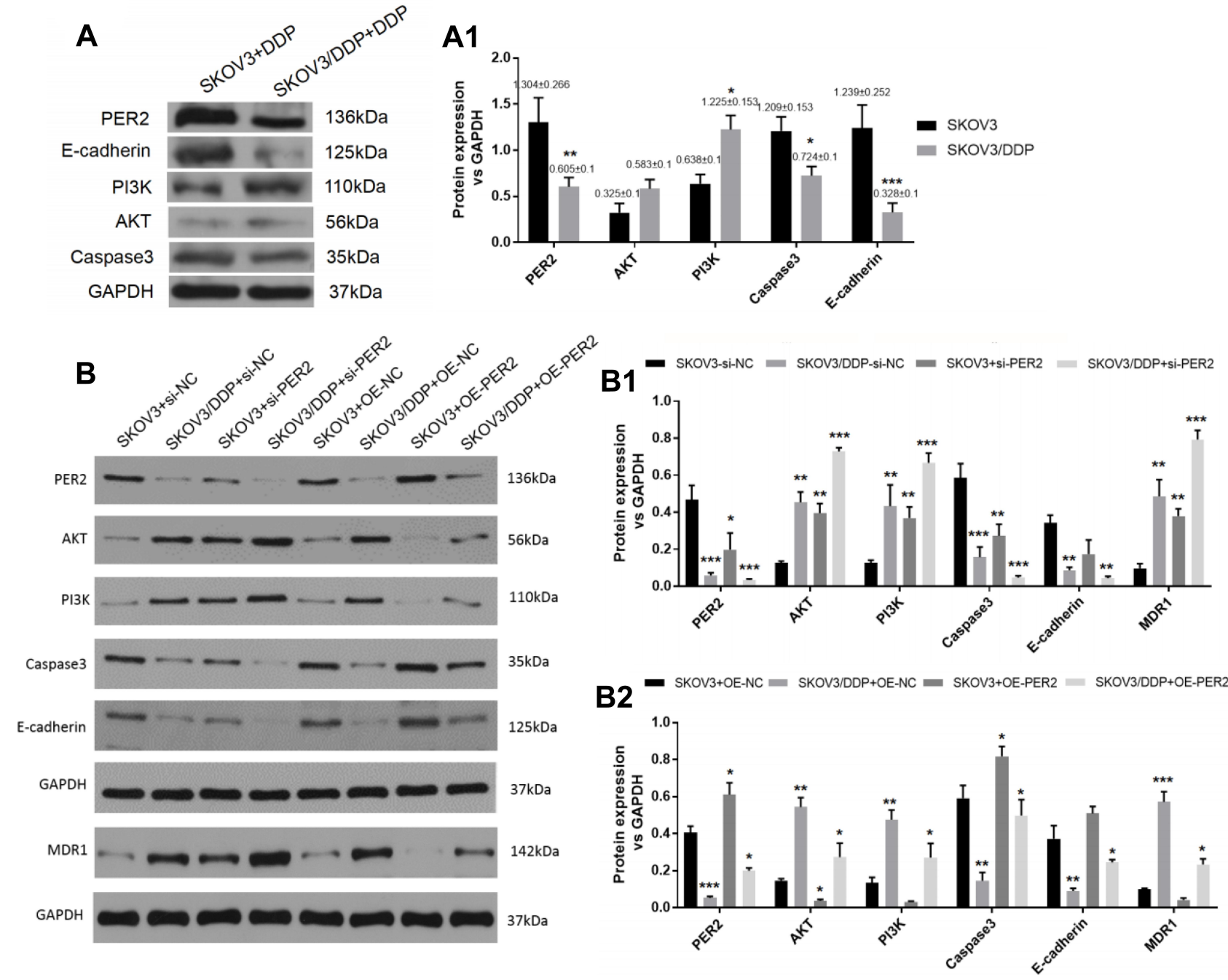

Figure 4 PER2 suppresses the PI3K/Akt pathway and MDRI while enhancing caspase-3 and E-cadherin protein expression in SKOV3 cells. Western blot was performed the protein levels of PER2, AKT, PI3K, caspase 3, and E-cadherin for SKOV3 and SKOV3/DDP cells, with GAPDH as an internal control, and quantification with Image-J. P value of PER2 was 0.007I, PI3K 0.027I, caspase 3 0.0399, E-cadherin 0.0005. (AI) The relative proteins expression of the two groups to (A), between SKOV3 and SKOV3/DDP cells. $* p<0.05,{ }^{*} p<0.01$, ${ }^{* *} p<0.00$ I, SKOV3/DDP vs SKOV3. (B) Western blot was performed at $48 \mathrm{~h}$ after treatment to determine the protein levels of PER2, AKT, $\mathrm{PI} 3 \mathrm{~K}$, caspase 3, E-cadherin, and MDRI for SKOV3 and SKOV3/DDP cells transfected with si-RNA or overexpressing vector, vs si-NC or empty vector, with GAPDH as an internal control. (B I-B2) The relative proteins expression of the eight groups to (B). ${ }^{*} p<0.05, * * p<0.01$, $* * * p<0.001$ vs OE-NC between SKOV3 and SKOV3/DDP cells. $*_{p}<0.05, * * p<0.01$, ***p $<0.001$, SKOV3/DDP-si-PER2, SKOV3/DDP-si-NC and SKOV3-si-PER2 vs SKOV3-si-NC. *p < 0.05, **p < 0.0I, ***p < 0.00I, SKOV3/DDP-OEPER2, SKOV3/DDP-OE-NC and SKOV3-OE-PER2 vs SKOV3-OE-NC. Data are mean \pm SD, $n=3$.

(Figure 4B). The gray value of Western blot was as shown in Figure 4B1-2.

Overexpression of PER2 significantly reversed the above alterations in both parental and DDP-resistant SKOV3 cells, whereas its knockdown showed opposite effects. In addition, PER2 knockdown further upregulated MDR1 that was significantly enhanced in SKOV3/DDP cells. In contrast, overexpression of PER2 significantly attenuated the protein expression of MDR1 in both cell lines.

Taken together, these data suggested that loss of PER2 might contribute to cisplatin resistance, possibly by activating EMT and PI3K/Akt signaling, inhibiting cisplatin- induced cell apoptosis, and promoting drug efflux. Overexpression of PER2 could reverse these alterations to restore cisplatin sensitivity.

\section{PER2 Enhances the Sensitivity of SKOV3/ DPP Cell-Derived Tumors to Cisplatin and Alleviates Systemic Inflammatory Response in Mice}

We next established a nude mouse xenograft model to further investigate the role of PER2 in cisplatin sensitivity. Figure 5A and $\mathrm{B}$ shows that PER2-overexpressing SKOV3/DPP cells yielded significantly reduced tumor 
masses compared with control cells during cisplatin treatment, indicating that PER2 increased the sensitivity of xenograft tumors to cisplatin. Figure 5C, IHC staining showed an apparent decrease in CD34 expression in OEPER2 groups of SKOV3/DDP cells. These results were consistent with the tumor volume (Figure 5A and B), and strongly implied that PER2 enhances the sensitivity of SKOV3/DPP cell-derived tumors to cisplatin.

PER2 overexpression also resulted in significantly decreased protein levels of PI3K, AKT, MDR1, and MRP1, but increased protein amounts of caspase-3, E-cadherin, and DYRK2 in tumor tissues (Figure 6A1), consistent with in vitro data (Figure 4A-B). The gray value of Western blot was as shown in Figure 6A2. To further explore whether PER2 modulates systemic inflammation to affect chemosensitivity, we detected the serum levels of TNF- $\alpha$ and IL- 6 in tumor-bearing mice before the initiation of treatment. Figure 6B-C shows that mice bearing PER2-overexpressing tumors had significantly lower serum levels of TNF- $\alpha$ and IL-6 compared with the OENC group. This finding suggested that PER2 overexpression might alleviate systemic inflammation to sensitize ovarian tumors to cisplatin treatment.

\section{Discussion}

The present study suggested that ectopic expression of PER2 sensitizes ovarian cancer cells to cisplatin possibly by modulating multiple mechanisms, serving as a promising therapeutic strategy to overcome chemoresistance in patients with ovarian cancer.

Consistent with recent studies associating loss of PER2 with chemoresistance in cancer, ${ }^{17,26,27}$ we found that SKOV3/DDP cells had dramatically decreased mRNA levels of PER2 compared with parental cells. Previous studies have shown that microRNAs, such as miR-24, miR-449a, and miR-484, inhibit PER2 transcription by binding to its $3^{\prime}$-untranslated region. ${ }^{28,29}$ Epigenetic mechanisms, including histone modifications and DNA

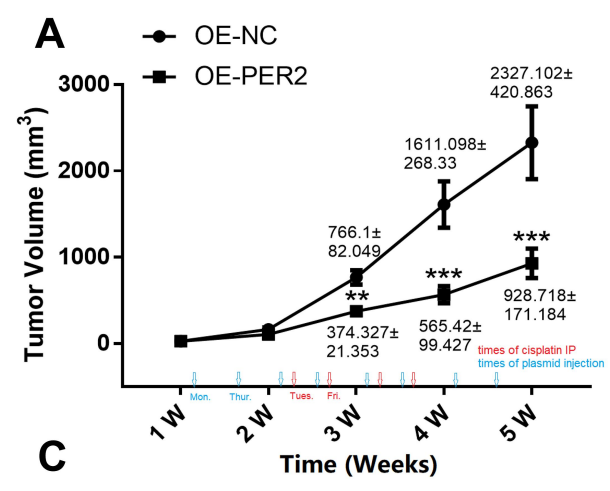

OE-PER2
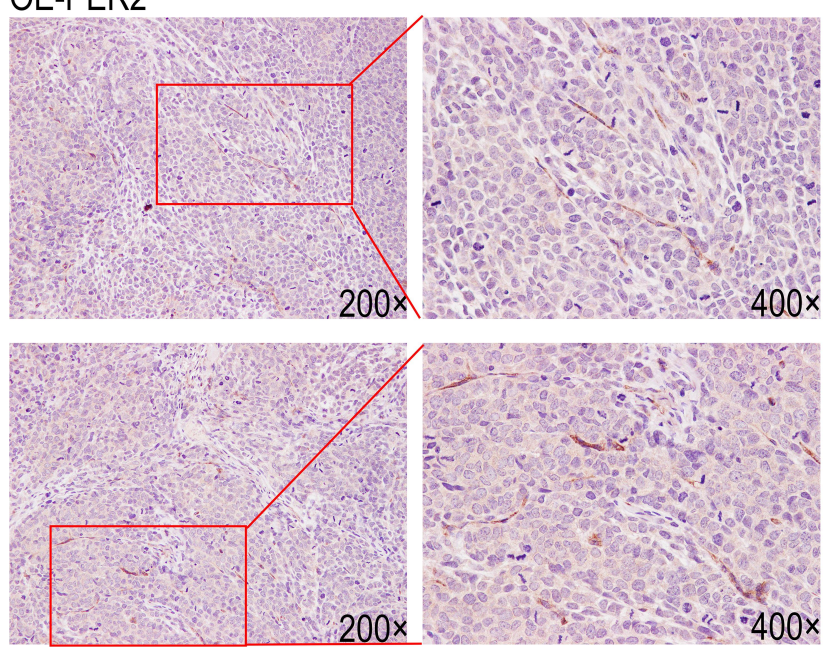

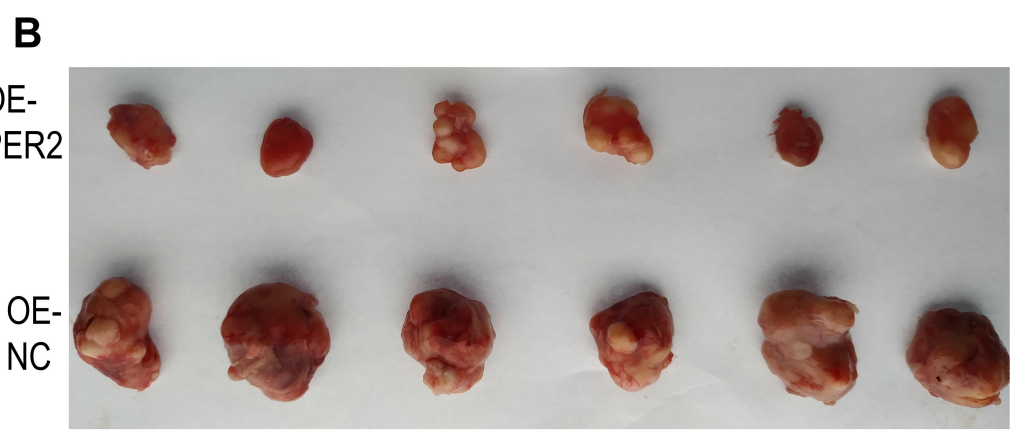

OE-NC
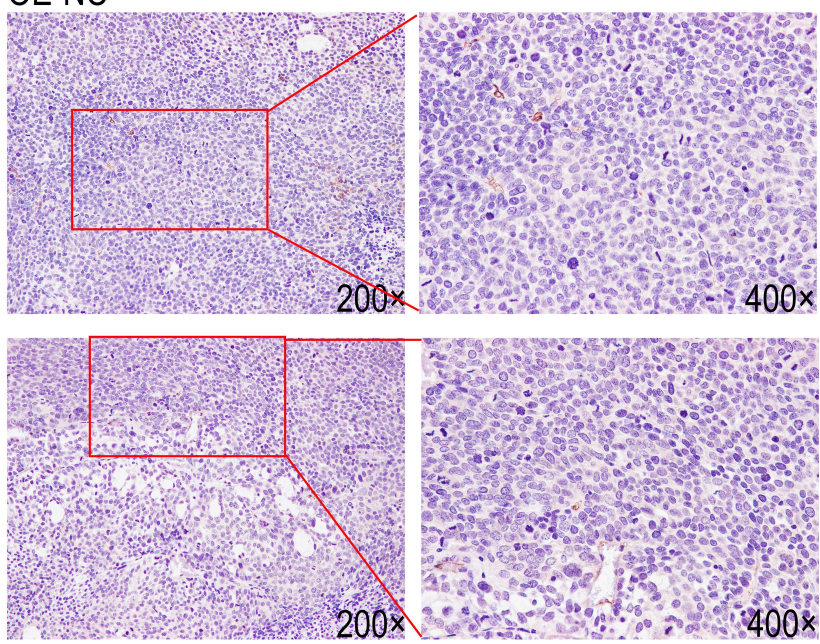

Figure 5 PER2 promotes the sensitivity of SKOV3/DPP cell-derived tumors to cisplatin in vivo. (A) Tumors were measured every week with a digital caliper, and tumor volume $(\mathrm{V})$ was derived by the following equation $V=\left(\right.$ length $\times$ width $\left.^{2}\right) / 2$. Data are mean $\pm S D$. $* * p<0.0$ I, $* * * p<0.00$ I, vs control; $n=5$. (B) Mice were sacrificed after 3 weeks of cisplatin treatment, and tumors were immediately removed and imaged. The tumor volume were more bigger than OE-NC group, vs OE-PER2. (C) IHC detection found that expression of CD34 were higher in OE-PER2 group. 

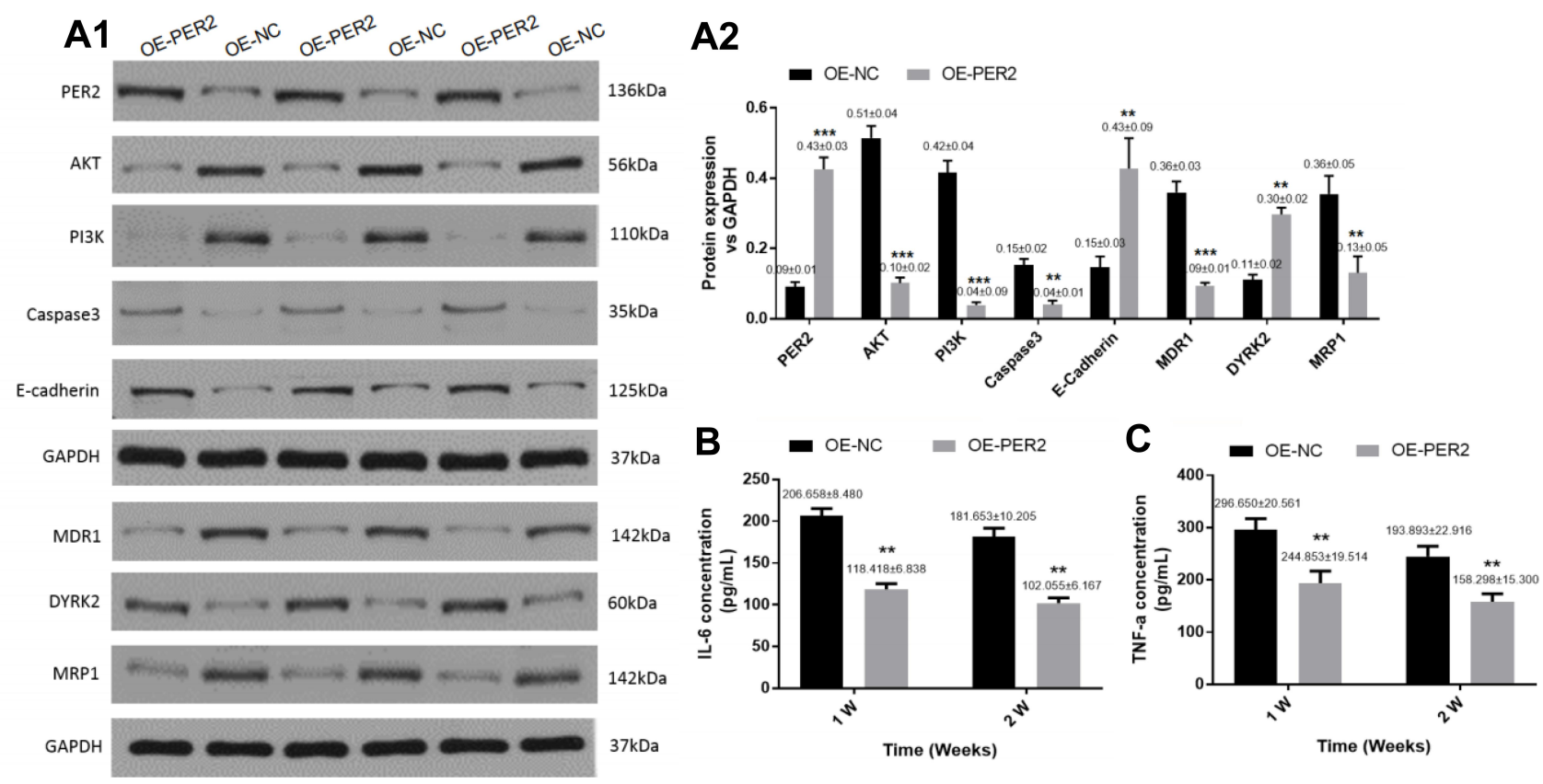

Figure 6 PER2 enhances the sensitivity of SKOV3/DPP cell-derived tumors to cisplatin and alleviates systemic inflammatory response in vivo. (AI) Tumor tissue samples (three repetitions in each group) were randomly selected from each group. Western blot was performed to determine the protein levels of PER2, AKT, PI3K, caspase 3, E-cadherin, MDRI, DYRK2, and MRPI in tumor tissue samples, with GAPDH as an internal control. (A2) The expression of PER2, Caspase 3, E-cadherin and DYRK2 in the tumor tissue injected with PER2 overexpression was higher than that in the tumor tissue injected with empty plasmid; the expression of Akt, PI3K, MDRI and MRPI in the tumor tissue injected with PER2 overexpression plasmid was lower than that in the tumor tissue injected with empty plasmid. Data are mean \pm SD. $* * p<0.0$ I, $* * * p<0.00$, OE-PER2 vs OE-NC; $n=5$. (B-C) The serum levels of IL- 6 and TNF- $\alpha$ were measured by ELISA at I and 2 weeks, respectively, post-tumor cell inoculation. P value of IL-6 was 0.0059 and 0.0032 at $I$ and 2 weeks, $P$ value of TNF- $\alpha$ was 0.0064 and 0.0022 at $I$ and 2 weeks. Data are mean \pm SD. $* * p<0.01$, OE-PER2 vs OE-NC; $n=5$.

methylation, also play important roles in regulating gene expression. It has been reported that sevoflurane anesthesia suppresses PER2 expression by reducing histone acetylation of the proximal region of the PER2 promoter. ${ }^{30}$ Hypermethylation of the PER2 promoter alters PER2 expression in glioma cells, increasing the survival of cancer cells and promoting carcinogenesis due to disrupted circadian clock. ${ }^{31}$ In this study, we found that SKOV3/ DDP cells had significantly increased promoter methylation at the nine $\mathrm{CpG}$ sites compared with parental cells, suggesting that $\mathrm{CpG}$ promoter methylation possibly contributes to loss of PER2 in SKOV3/DDP cells.

Previous studies have reported PI3K/Akt pathway activation in different cisplatin-resistant cancer cells. ${ }^{25,32}$ Similarly, the above results showed that SKOV3/DDP cells had significantly increased protein levels of PI3K and Akt compared with parental cells. Once activated, Akt phosphorylates Bcl-2-associated death protein (BAD) on ser-136 and facilitates its dissociation from the Bcl-2/ Bcl-X complex, thereby suppressing the apoptosisinducing function of BAD. ${ }^{33}$ Cisplatin treatment induces BAD phosphorylation at ser-112 via Erk and at ser-136 via PI3K/Akt, protecting cancer cells from apoptosis. ${ }^{34}$ However, the mechanisms underlying cisplatin-induced activation of $\mathrm{PI} 3 \mathrm{~K} / \mathrm{Akt}$ remains largely unknown. A recent study assessing A549/DDP cells showed that PER2 knockdown activates, whereas its overexpression inhibits the PI3K/AKT pathway. ${ }^{35}$ In agreement, we found that PER2 reduced the protein levels of PI3K and Akt in SKOV3/DDP cells and SKOV3 cell-derived tumor tissues. These findings suggest that PER2 is an upstream suppressor of $\mathrm{PI} 3 \mathrm{~K} / \mathrm{Akt}$, and can reverse PI3K/Aktmediated cisplatin resistance in cancer.

Recent studies have shown that PER2 suppresses cancer cell proliferation while promoting apoptosis in oral squamous cell carcinoma, osteosarcoma, lung adenocarcinoma, and liver carcinoma. ${ }^{35-38}$ PER2 overexpression downregulates Bcl-2 while increasing the expression of cleaved caspase 3 in osteosarcoma cells. ${ }^{37}$ Similarly, overexpression of mouse PER2 also downregulates Bcl-XL and Bcl-2 while upregulating Bax in mouse lung carcinoma cells. ${ }^{39}$ Consistent with these findings, the current results showed that PER2 could enhance the protein expression of caspase 3 and promote apoptosis, which was suppressed in SKOV3/DDP cells. Xenograft tumors derived from PER2-overexpressing SKOV3 cells exhibited increased sensitivity to cisplatin and enhanced expression of caspase 3 compared with those from parental cells. 
These results suggest that PER2 increases cisplatin sensitivity and reverses cisplatin resistance by inducing apoptotic cell death in ovarian cancer.

Treatments with TNF $\alpha$ and IL-6 induce platinum resistance in ovarian cancer cell lines by upregulating MDR1. ${ }^{14}$ Our in vivo data showed that mice bearing PER2overexpressing tumors had significantly lower serum levels of TNF- $\alpha$ and IL-6 compared with the control group before the initiation of cisplatin treatment. This finding suggests that PER2 overexpression might alleviate systemic inflammation to sensitize ovarian tumors to cisplatin. We also found that PER2 significantly reduced the protein levels of MDR1 and MRP1 in both SKOV3 and SKOV3/DDP cells, which has not been reported elsewhere. This finding suggests that PER2 increases the sensitivity of ovarian cancer cells to cisplatin, possibly by reducing drug transporter-mediated drug efflux.

\section{Conclusion}

In summary, loss of PER2, accompanied by PI3K/Akt upregulation and caspase 3 and E-cadherin downregulation, is associated with cisplatin resistance in ovarian cancer cells. PER2 overexpression reverses these alterations and sensitizes ovarian cancer cells to cisplatin both in vitro and in vivo, constituting a potential therapeutic strategy for overcoming cisplatin resistance in ovarian cancer. Circadian rhythm is involved in the development of drug resistance in ovarian cancer. It is suggested that according to the difference of biological clock mechanism between normal cells and cancer cells, chemotherapy with circadian rhythm may reduce the phenomenon of chemotherapy resistance, improve the curative effect and improve the clinical prognosis of patients.

\section{Abbreviations}

PER2, period circadian regulator 2; EMT, epithelialmesenchymal transition; PI3K, phosphatidylinositol 3-kinase; Akt, protein kinase B; MDR1, multidrug resistance 1; MRP1, MDR1-related protein 1; TNF- $\alpha$, tumor necrosis factor-alpha; DYRK2, Dual-specificity tyrosinephosphorylation-regulated kinase 2; IL-6, interleukin 6; PBS, phosphate-buffered saline; BAD, Bcl-2-associated death protein; SKOV3 cells Human epithelial ovarian cancer SKOV3 (parental) cells; SKOV3/DDP cells cisplatin-resistant SKOV3 cells; ELISA, enzyme-linked immunosorbent assay; IHC, immunohistochemistry; CCK-8, cell counting kit-8; qRT-PCR quantitative real-time PCR; $\mathrm{SD}$, standard deviation; si-RNA, small interfering RNA;
DDP, cisplatin-resistant; NC, negative control; OE, overexpressing.

\section{Data Sharing Statement}

The datasets used and/or analysed during the current study are available from the corresponding author on reasonable request.

\section{Ethics Approval and Consent to Participate}

This study was approved by the Ethics Committee of SHANXI MEDICAL UNIVERSITY.

\section{Consent for Publication}

Not applicable.

\section{Author Contributions}

All authors made a significant contribution to the work reported, whether that is in the conception, study design, execution, acquisition of data, analysis and interpretation, or in all these areas; took part in drafting, revising or critically reviewing the article; gave final approval of the version to be published; have agreed on the journal to which the article has been submitted; and agreed to be accountable for all aspects of the work.

\section{Funding}

The study was supported by the Natural Science Foundation of Shanxi Province (NO. 201701D221275). The funder provides part of the research funding. However, it does not participate in the research and the completion of the manuscript.

\section{Disclosure}

The authors declare that they have no competing interests.

\section{References}

1. Coburn SB, Bray F, Sherman ME, Trabert B. International patterns and trends in ovarian cancer incidence, overall and by histologic subtype. Int J Cancer. 2017;140:2451-2460. doi:10.1002/ijc.30676

2. Dinkelspiel HE, Champer M, Hou J, et al. Long-term mortality among women with epithelial ovarian cancer. Gynecol Oncol. 2015;138:421-428. doi:10.1016/j.ygyno.2015.06.005

3. Pokhriyal R, Hariprasad R, Kumar L, Hariprasad G. Chemotherapy resistance in advanced ovarian cancer patients. Biomark Cancer. 2019;11:1179299X19860815. doi:10.1177/1179299X19860815

4. Dasari S, Tchounwou PB. Cisplatin in cancer therapy: molecular mechanisms of action. Eur J Pharmacol. 2014;740:364-378. doi:10.1016/j.ejphar.2014.07.025

5. Kelland L. The resurgence of platinum-based cancer chemotherapy. Nat Rev Cancer. 2007;7:573-584. doi:10.1038/nrc2167 
6. Ledermann JA. First-line treatment of ovarian cancer: questions and controversies to address. Ther Adv Med Oncol. 2018;10:1758835918 768232. doi:10.1177/1758835918768232

7. Li SS, Ma J, Wong AST. Chemoresistance in ovarian cancer: exploiting cancer stem cell metabolism. J Gynecol Oncol. 2018;29:e32. doi:10.3802/jgo.2018.29.e32

8. Chang AM, Duffy JF, Buxton OM, et al. Chronotype genetic variant in PER2 is associated with intrinsic circadian period in humans. Sci Rep. 2019;9:5350. doi:10.1038/s41598-019-41712-1

9. Hamilton JP. Epigenetics: principles and practice. Dig Dis. 2011;29:130-135. doi:10.1159/000323874

10. Sun CM, Huang SF, Zeng JM, et al. Per2 inhibits k562 leukemia cell growth in vitro and in vivo through cell cycle arrest and apoptosis induction. Pathol Oncol Res. 2010;16:403-411. doi:10.1007/s12253009-9227-0

11. Cheng AY, Zhang Y, Mei HJ, et al. Construction of a plasmid for overexpression of human circadian gene period2 and its biological activity in osteosarcoma cells. Tumour Biol. 2015;36:3735-3743. doi:10.1007/s13277-014-3013-7

12. Zhanfeng N, Chengquan W, Hechun X, et al. Period2 downregulation inhibits glioma cell apoptosis by activating the MDM2-TP53 pathway. Oncotarget. 2016;7:27350-27362. doi:10.18632/oncotarget.8439

13. Wang Z, Li L, Wang Y. Effects of Per2 overexpression on growth inhibition and metastasis, and on MTA1, nm23-H1 and the autophagy-associated $\mathrm{PI} 3 \mathrm{~K} / \mathrm{PKB}$ signaling pathway in nude mice xenograft models of ovarian cancer. Mol Med Rep. 2016;13:4561-4568. doi:10.3892/mmr.2016.5116

14. Matassa DS, Amoroso MR, Lu H, et al. Oxidative metabolism drives inflammation-induced platinum resistance in human ovarian cancer. Cell Death Differ. 2016;23:1542-1554. doi:10.1038/cdd.2016.39

15. Rodon J, Dienstmann R, Serra V, Tabernero J. Development of PI3K inhibitors: lessons learned from early clinical trials. Nat Rev Clin Oncol. 2013;10:143-153. doi:10.1038/nrclinonc.2013.10

16. Tan J, You Y, Xu T, et al. Par-4 downregulation confers cisplatin resistance in pancreatic cancer cells via PI3K/Akt pathway-dependent EMT. Toxicol Lett. 2014;224:7-15. doi:10.1016/j.toxlet.2013.10.008

17. Carden CP, Stewart A, Thavasu P, et al. The association of PI3 kinase signaling and chemoresistance in advanced ovarian cancer. Mol Cancer Ther. 2012;11:1609-1617. doi:10.1158/1535-7163.MCT-11-0996

18. Gloushankova NA, Rubtsova SN, Zhitnyak IY. Cadherin-mediated cell-cell interactions in normal and cancer cells. Tissue Barriers. 2017;5:e1356900. doi:10.1080/21688370.2017.1356900

19. Haslehurst AM, Koti M, Dharsee M, et al. EMT transcription factors snail and slug directly contribute to cisplatin resistance in ovarian cancer. BMC Cancer. 2012;12:91. doi:10.1186/1471-2407-12-91

20. Robey RW, Pluchino KM, Hall MD, Fojo AT, Bates SE, Gottesman MM. Revisiting the role of ABC transporters in multidrug-resistant cancer. Nat Rev Cancer. 2018;18:452-464. doi:10.1038/s41568-018-0005-8

21. Schondorf T, Neumann R, Benz C, et al. Cisplatin, doxorubicin and paclitaxel induce mdr1 gene transcription in ovarian cancer cell lines. Recent Results Cancer Res. 2003;161:111-116. doi:10.1007/9783-642-19022-3_10

22. Beretta GL, Benedetti V, Cossa G, et al. Increased levels and defective glycosylation of MRPs in ovarian carcinoma cells resistant to oxaliplatin. Biochem Pharmacol. 2010;79:1108-1117. doi:10.1016/j. bcp.2009.12.002

23. Savant SS, Sriramkumar S, O'Hagan HM. The role of inflammation and inflammatory mediators in the development, progression, metastasis, and chemoresistance of epithelial ovarian cancer. Cancers (Basel). 2018;10. doi:10.3390/cancers10080251
24. Wang Y, Niu XL, Qu Y, et al. Autocrine production of interleukin-6 confers cisplatin and paclitaxel resistance in ovarian cancer cells. Cancer Lett. 2010;295:110-123. doi:10.1016/j.canlet.2010.02.019

25. Wang LG. Regulation of response to radiotherapy by $\beta$-arrestin 1 in Non-small cell lung cancer. J Cancer. 2019;10(17):4085-4095. doi:10.7150/jca.30012

26. Zhang Y, Bao C, Mu Q, et al. Reversal of cisplatin resistance by inhibiting PI3K/Akt signal pathway in human lung cancer cells. Neoplasma. 2016;63:362-370. doi:10.4149/304 150806N433

27. Katamune C, Koyanagi S, Hashikawa KI, et al. Mutation of the gene encoding the circadian clock component PERIOD2 in oncogenic cells confers chemoresistance by up-regulating the Aldh3a1 gene. $J$ Biol Chem. 2019;294:547-558. doi:10.1074/jbc.RA118.004942

28. Yoo SH, Kojima S, Shimomura K, et al. Period2 3'-UTR and microRNA-24 regulate circadian rhythms by repressing PERIOD2 protein accumulation. Proc Natl Acad Sci U S A. 2017;114:E8855E64. doi:10.1073/pnas.1706611114

29. Wang Y, Lv K, Chen H, et al. Functional annotation of extensively and divergently expressed miRNAs in suprachiasmatic nucleus of Clock (Delta19) mutant mice. Biosci Rep. 2018:38. doi:10.1042/ BSR20180233.

30. Mori K, Iijima N, Higo S, et al. Epigenetic suppression of mouse Per2 expression in the suprachiasmatic nucleus by the inhalational anesthetic, sevoflurane. PLoS One. 2014;9:e87319. doi:10.1371/journal.pone.0087319

31. Fan W, Chen X, Li C, et al. The analysis of deregulated expression and methylation of the PER2 genes in gliomas. J Cancer Res Ther. 2014;10:636-640. doi:10.4103/0973-1482.138202

32. Peng DJ, Wang J, Zhou JY, Wu GS. Role of the Akt/mTOR survival pathway in cisplatin resistance in ovarian cancer cells. Biochem Biophys Res Commun. 2010;394:600-605. doi:10.1016/j. bbrc.2010.03.029

33. Sakamaki J, Daitoku H, Ueno K, Hagiwara A, Yamagata K, Fukamizu A. Arginine methylation of BCL-2 antagonist of cell death (BAD) counteracts its phosphorylation and inactivation by Akt. Proc Natl Acad Sci U S A. 2011;108:6085-6090. doi:10.1073/ pnas. 1015328108

34. Hayakawa J, Ohmichi M, Kurachi $\mathrm{H}$, et al. Inhibition of BAD phosphorylation either at serine 112 via extracellular signal-regulated protein kinase cascade or at serine 136 via Akt cascade sensitizes human ovarian cancer cells to cisplatin. Cancer Res. 2000;60:5988-5994.

35. Chen B, Tan Y, Liang Y, et al. Per2 participates in AKT-mediated drug resistance in A549/DDP lung adenocarcinoma cells. Oncol Lett. 2017;13:423-428. doi:10.3892/ol.2016.5430

36. Wang Q, Ao Y, Yang K, Tang H, Chen D. Circadian clock gene Per2 plays an important role in cell proliferation, apoptosis and cell cycle progression in human oral squamous cell carcinoma. Oncol Rep. 2016;35:3387-3394. doi:10.3892/or.2016.4724

37. Qin T, Lu XT, Li YG, et al. Effect of Period 2 on the proliferation, apoptosis and migration of osteosarcoma cells, and the corresponding mechanisms. Oncol Lett. 2018;16:2668-2674. doi:10.3892/ ol.2018.8952

38. Mteyrek A, Filipski E, Guettier C, Okyar A, Levi F. Clock gene Per2 as a controller of liver carcinogenesis. Oncotarget. 2016;7:85832-85847. doi:10.18632/oncotarget.11037

39. Hua H, Wang Y, Wan C, et al. Circadian gene mPer2 overexpression induces cancer cell apoptosis. Cancer Sci. 2006;97:589-596. doi:10.1111/j.1349-7006.2006.00225.x 


\section{Publish your work in this journal}

Cancer Management and Research is an international, peer-reviewed The manuscript management system is completely online and includes open access journal focusing on cancer research and the optimal use of a very quick and fair peer-review system, which is all easy to use. preventative and integrated treatment interventions to achieve improved Visit http://www.dovepress.com/testimonials.php to read real quotes outcomes, enhanced survival and quality of life for the cancer patient. from published authors.

Submit your manuscript here: https://www.dovepress.com/cancer-management-and-research-journal 